\title{
Organization Revealed by Recall Orders and Confirmed by Pauses
}

\author{
Judith S. Reitman AND Henry H. Rueter \\ The University of Michigan
}

\begin{abstract}
The technique introduced here induces the organization of information in memory from systematic inspection of regularities in free recall. The form of the representation of this organization is an "ordered tree." The technique has the advantage of being based on a theory of the way in which the data were generated and can be shown to produce a unique structure that captures all the kinds of regularities the theory of recall prescribes. Also presented is a collateral technique for measuring the amount of organization evidenced in a struture, as well as a procedure for identifying errors. The experimental work shows the technique's ability to recover the details of an organization presented to subjects and provides converging evidence for the particular structures induced from the pattern of recall pauses. In addition, the application of the technique to structures unknown a priori produced organizations that were easy to interpret and a second set of pauses that further confirmed the details of the induced structures.
\end{abstract}

A number of contemporary topics in cognitive psychology involve investigation of complexly organized knowledge bases and the mental processes that operate on them. For example, researchers in human factors engineering are concerned with building man-machine interfaces that are compatible with the knowledge the user brings to the situation. Researchers in problem solving examine the changes in knowledge structures and mental operations that occur as one moves from the novice to the expert level. And, educators attempt to devise instructional programs that build complexly organized knowledge bases in the student. Essential to all of these investigations is a description of the form and the details of the psychological representation of the knowledge base. This paper introduces a technique that provides such a concrete representation of some aspects of the mental organization of complex categories, based on inferences from multitrial free recall.

There are a number of other techniques that infer details of a subject's mental organization. Selecting those that are appropriate for a particular investigation depends on a number of issues: the kind of behavior under investigation (e.g., similarity judgments, confusions, the order in which

This research was supported by NSF grant BNS-76-82806 to the first author. We wish to thank J. E. Keith Smith and Kenneth Guire for their helpful advice concerning statistical analyses of the pause data. Requests for reprints should be sent to Professor Judith S. Reitman, Human Performance Center, 330 Packard, Ann Arbor, MI 48104. 
items are recalled, recall pauses, etc.), the form that the inferred organization is to take (e.g., a configuration in $n$-dimensional space, a rooted or unrooted tree, a set of overlapping clusters, etc.), and, most important, the kinds of questions one wants answered (e.g., questions about graded properties of the objects, about which items function together as a chunk, etc.).

The technique most commonly used for inferring the mental organization of a set of items is multidimensional scaling (Shepard, 1962a, b; Kruskal, 1964a, b; Cunningham \& Shepard, 1974). This technique begins with a matrix of psychological distances, which may be obtained from any one of a number of tasks: direct similarity judgments of all pairs of objects, frequencies with which pairs of items are sorted into the same category, discriminative reaction times, confusion errors, or average number of items recalled between two items in a free recall task, to name a few. The result is a configuration of the objects in $n$-dimensional space that preserves individual pair-wise distances as closely as possible. The investigator's task is to interpret the dimensions and to make inferences about the relative distances among the objects according to the purpose of the study, e.g., which of a set of animal names is most central to a particular cluster (most prototypical), or which vowel sound is most likely to be confused with what other sounds, etc.

Because a multidimensional scaling configuration is located in a continuous space, this technique best answers questions about graded properties (e.g., prototypicality, strength of an association) of items, properties that are expected to vary continuously. It easily represents judgments about physical dimensions such as brightness, shape, or numerical magnitude, but may not be as illuminating about objects that are expected to form discrete clusters. A good example of this point is shown in the work of Sattath and Tversky (1977), in which a multidimensional scaling configuration of animal names (well-organized taxonomic categories) had dimensions and distances that were difficult to interpret, whereas a hierarchical configuration had clusters and nestings that were easily identified from known relationships between the objects. In fact, Holman (1972) has made this dichotomy explicit, showing that if distances perfectly fit the assumptions of hierarchical clustering, they are necessarily incompatible with the assumptions of multidimensional scaling, and vice versa.

A number of theoretical advances have provided analyses of distance matrices that result in more discrete configurations. To mention a few, Johnson's (1967) hierarchical clustering scheme produces a tree with clustering level indicating distances between objects; Cunningham's (Note 1) analysis produces a "weighted free tree," a graph structure in which distances are proportional to the path length between two objects; and Shepard and Arabie's method (1979) produces a configuration of 
overlapping clusters that is typically represented by closed curves around items in a two-dimensional scaling solution.

One clear advantage of all these techniques is that they start with an atheoretical distance matrix, a very rich set of data that can be derived from a number of tasks. There is, however, an attendant disadvantage to this generality of application. Often these techniques are applied mechanically, regardless of the origin of the psychological distances, whether they come from confusions or recall orders. Moreover, any analysis of a distance matrix is already several steps away from the data and from regularities-perhaps inexpressible in a distance matrix - that might reveal important properties of the mental processes that produced them.

Two further problems plague analysis of distance matrices. First, distances derived from direct similarity judgments are limited in reliability; they change with the context in which the particular items are judged (Arnold, 1971). Second, analyses of distance matrices usually require that the distances by symmetric. Yet, in some cases the psychological processes involved produce distances that are clearly asymmetric. The final configuration must then fail to capture the potentially important kind of regularity.

The results from Friendly's (1977) use of Johnson's hierarchical clustering scheme on free recall data is such an example. Friendly filled a half-matrix with symmetric distances derived from multitrial free recall data, where distance was a function of the average number of items recalled between two items, regardless of the order of recall. But there is a lot of ordering in free recall. The recall processes that generate the data are likely to produce asymmetric distances. Given that some items in memory are organized as a list, $a \rightarrow b \rightarrow c \rightarrow d$, the distance from an item outside this list to $d$ is going to be large (in that the subject probably recalled $a, b$, and $c$ before getting to $d$ ), but the distance from $d$ to that outside item is going to be very short. Such directional organization in recall (recalling some items as short lists) is evidenced so often that early measures of the amount of subjective organization (e.g., Tulving, 1962) were based solely on them (see Shuell, 1969, for a review). Graesser (Note 3) and Jantz and Underwood (1958) have shown a second form of asymmetry in free recall: The ability of a cue to elicit recall of an item is not always the same as that of the item for the recall of the cue. Associations are often asymmetric, and an investigator who uses an analytical 
further difficulty for the investigator trying to make reliable inferences about the "true" psychological representation.

Recently, behaviors other than those easily converted to "distances" have been examined with an eye toward inducing the underlying organization of information in memory. Since the order of recall seems clearly to reflect underlying organization (as in "free association"), many have focused on regularities in recall. For example: (1) Because subjects often alternate series of correct responses and errors in learning a serial list, Bower and Winzenz (1969) and Martin and Noreen (1974) defined serial cluster (or chunk) boundaries as those points at which many anticipation errors occur during learning; (2) Because pauses in recall occur at boundaries of experimenter-defined chunks (Bower \& Springston, 1970; McLean \& Gregg, 1967; Gelfand, Note 2), Chase and Simon (1973a, b) associated chunk boundaries with long pauses; (3) Bushke (1976) developed a "2-D" recall method in which he asks each subject to indicate his introspecive organization by writing associated clusters of items near each other on a recall sheet; (4) Similarly, Reitman (1976) and Egan and Schwartz (1979) asked their subjects to reveal their organizations by drawing closed curves around those elements that they felt were functionally related.

Although these techniques use behaviors that are clearly related to the underlying organization, they have not evolved into the most useful kind of representation. The data are typically unreliable: Pauses are highly variable (see Reitman, 1976) and introspective reports can change with the subject's perception of the intent of the inquiry. Furthermore, the representations typically include only one level of structure, although Reitman's drawn enclosures technique did indicate some overlapping clusters and some nestings.

Recently, more precise, sophisticated techniques specifically intended for free recall data have been developed. These techniques differ from those discussed since they scrutinize the set of recall orders itself, not a derived distance matrix. They differ from multidimensional scaling in particular on two important aspects as well: They produce a hierarchical-not spatial-representation, and thus answer questions about discrete-not graded-properties of the data, such as which items function together as a chunk in memory and perception.

These techniques have also been developed by two investigators outside our own laboratory: Monk (1976) and Vorberg (Note 5). All three techniques are based on the premise that subjects proceed very regularly when recalling complexly organized material, and recall all elements of one chunk before moving to the next. The techniques capitalize on this regularity by inspecting the strings of items produced in free recall for chunks, which are defined as sets of items that are consistently recalled 
together without other items intervening. Although both the Monk and Vorberg algorithms share the spirit of our technique, they are deficient in subtle, but important, respects. Both analyze recall strings "bottom up." Each chunk is identified and labelled by a symbol; the recall strings are rewritten with the symbols replacing the chunked elements, and then examined for second-level chunks. Again, these strings are rewritten with new symbols representing the second level chunks and are then reexamined for third-level chunks, and so on. Eventually, all strings will be rewritten as a single symbol. The process is then reversed to display a chunk hierarchy.

Vorberg uses rewriting without regard for the items within a chunk, ${ }^{1}$ while Monk's algorithm rightly recognizes two kinds of directionality in chunks. Distinctions are made between those chunks whose constituents (either items or other chunks) have always been recalled in the same order (unidirectional), those whose constituents were recalled in one order or its inverse (bidirectional), and those in which items were recalled in a variety of mixed orders (nondirectional). Monk's algorithm is, in fact, the one we began our investigation with. Our subsequent mathematical investigation, involving the application of lattice theory to this problem, has shown that when chunks are identified "bottom up," even with directionality incorporated, the resulting tree may not be independent of the order of examination of strings. Thus, even Monk's algorithm may not find all the structure in the strings, since premature rewriting will prevent recognition of chunks at higher levels. Our algorithm proceeds "top down," to produce both a unique, complete solution and to identify higher order directionality that Monk's algorithm can miss. Monk claims that his algorithm captures all the regularities of chunking and directionality evidenced in the data (the "most parsimonious representation") when in fact it does not. ${ }^{2}$

In the following section, we introduce a technique for inducing the organization of discrete information in memory from a set of free recall orders. The technique, based on a theory of free recall, produces a unique structure that captures important regularities in the data-all those predicted by the theory of recall. In addition, a measure of the amount of

\footnotetext{
${ }^{1}$ Given the recall strings $B-Q-M-C-J$ and $C-J-M-Q-B$, the Vorberg algorithm will induce $(((B Q) M)(C J))$ if it searches the strings in the listed order and $((C J)(M Q) B)$ if it searches them in the opposite order. The meaning of the parenthesis notation can be found later in this paper.

${ }^{2}$ Given the same recall strings, $\mathrm{B}-\mathrm{Q}-\mathrm{M}-\mathrm{C}-\mathrm{J}$ and $\mathrm{C}-\mathrm{J}-\mathrm{M}-\mathrm{Q}-\mathrm{B}$, the Monk algorithm designates the structure as $((B Q M)[C J])$, and misses the chunks QMCJ and MCJ. Our algorithm finds $\langle\mathrm{BQM}[\mathrm{CJ}]\rangle$. The Monk structure has a PRO of $\ln (4)$, while ours has a PRO of $\ln (2)$. Premature rewriting of $B Q M$ in the first step of the Monk algorithm precluded recognition of the chunks QMCJ and MCJ.
} 
structure in a hierarchy is introduced, along with an important procedure that allows detection of errors. Our experimental work focuses on validation; the free recall strings of subjects taught a particular organization were analyzed, and their trees were compared with the structure presented. These same subjects were tape recorded while they recalled, allowing us a look at the correspondence between the subjects' induced structures and the pattern of pauses, and providing converging evidence for the veridicality of the induction technique. In addition, the technique was applied to information for which the organization was not known a priori, to show face validity in a more natural setting.

\section{The Technique}

An important part of the development of this technique involves theorizing about the processes that direct the subject's free recall. The data are examined for the kind of regularities these processes would produce, and the underlying structure is inferred from them. It is important to note at the outset that, regardless of the merits of our particular recall model, the technique produces a concise description of persistent chunklike regularities in the data, regularities that must be explained by any theory of recall.

The fundamental assumptions of our free recall model are that the items are organized into chunks and that the subject recalls chunks as units, recalling all of one chunk before proceeding to the next. This set of assumptions, more specifically, concerns the mental organization of these chunks and the mental processes that operate on their structure to produce the chunk patterns observed in free recall strings. ${ }^{3}$ Following Johnson (1972), we assume the chunks are mentally organized into a hierarchical tree whose terminal nodes represent the to-be-recalled items and nonterminal nodes represent mental codes for their constituents. The recall process (described by a traversal rule) operates on this hierarchy to produce recall orders. We extend this tree model of recall by assuming that traversal of the structure can be constrained by directionality at the nodes; the mental structure is thus called an "ordered tree." The constituents of some chunks (unidirectional chunks) can be accessed in only one order, as in reciting the alphabet. Others (bidirectional chunks) can be accessed in a single order or its reverse, as in counting up to or down from ten. Still others (nondirectional chunks) can be accessed in any order. As in the ordered tree of Fig. 1, unidirectional chunks are marked by singleheaded arrows over the constituent links (as in node 3), bidirectional chunks by double-headed arrows (as in node 1), and nondirectional chunks by the absence of an arrow of either kind (as in nodes 2, 5, and 4).

\footnotetext{
${ }^{3}$ See Discussion section for an examination of the consequences if either or both of these basic assumptions is violated.
} 


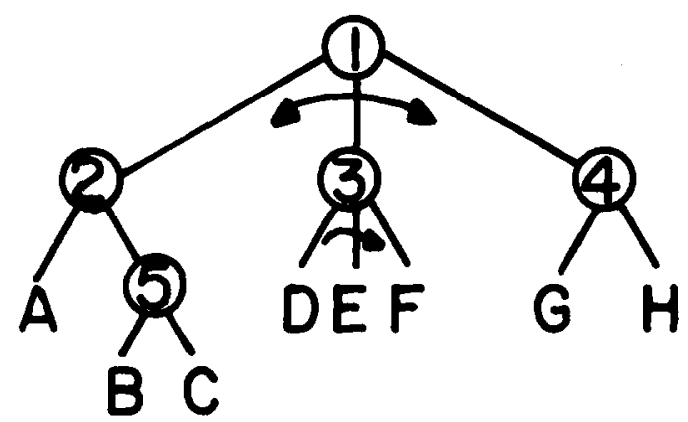

FIG. 1. An example of an ordered tree over the items labelled A through $H$.

In free recall the subject traverses an ordered tree, beginning at the root node, and descends the nodes until a terminal item is reached. Upon recalling that item, he moves up to its immediate superior node and descends its constituent links until all of its descendent terminal items have been recalled. The order of processing of the constituents of any node must, of course, be consistent with its order type. Once all of that node's descendents have been recalled, traversal is resumed at the immediately superior node, and each of its constituents are descended until all of its terminal items have been output. Traversal continues in this fashion until all items of the required set have been recalled. There is only a limited number of recall orders that can be produced under these traversal constraints, and the terminal items subsumed by any node will appear as a chunk in each of them.

Consider the example tree shown in Fig. 1 that represents a subject's presumed mental organization of items $A-H$. The recall rule prescribes that if the subject begins recall with item $B$, he will then recall $C$ (completing chunk 5), then item A (completing chunk 2), then D-E-F and $G-H$. Or if the subject begins with $G$, he will then recall $H$ (completing 4), then $D-E-F$ (completing 3 ) then either $A$ then $B$ and $C$, or $B$ and $C$ then A (completing 5 before 2 ). There are exactly 16 linear orders of the objects that can be produced by such traversals, and they all preserve the essential chunk structure inherent in the tree.

The technique is applied to data in the form of a set of complete recall orders. ${ }^{4}$ To collect appropriate data, subjects are asked to recall a large,

\footnotetext{
${ }^{4}$ Completeness of trials is crucial for this technique. When data are missing the investigator has several choices. One is to look at only those items that are recalled on all trials, appending incompletely recalled items to the resulting structure as "leaves" at points where the data indicate. Another is to modify the algorithm's search for chunks so that omissions are simply ignored rather than considered as chunk-violations. The former choice is preferable because the second is extremely expensive (the number of candidate chunks to be tested grows enormously) and there is no guarantee that the solution obtained is unique (i.e., it may depend on the order in which candidate groups are tested).
} 
well-learned set of items many times from many different starting points. It is important that the data include no omissions or intrusions, and that the subject give us a sample of the variety of orders he can produce. To induce variety, on some trials the subject is asked to recall freely (i.e., start with any item he chooses) and on other trials he is required to start with a "cue" item and those that "go with it." This cueing breaks any recall stereotypy that may build up in a session, and encourages variety.

From this set of cued and noncued recall strings, the algorithm efficiently finds the set of all chunks and represents this set as an ordered tree. In particular, the algorithm recursively examines the strings "top down" for chunks. The set of all such chunks forms a lattice which is then converted to a tree, with directionality indicated where appropriate. ${ }^{5}$ Equivalently, it can be written in the form of an expression with parentheses designating nondirectional chunks, square brackets unidirectional chunks, and angle brackets bidirectional chunks.

An important detail of this technique involves appropriate analysis of the cued trials. Since the cue item may be part of a chunk whose traversal is disrupted by the cueing process (directional chunks are particularly vulnerable), only that part of a cued trial that is assumed undisrupted should be analyzed. The disrupted and undisrupted segments of recall strings are identified in an initial step of the algorithm. First, the highestlevel disjoint chunks-formed by the subtrees of the root of the tree induced by all recall orders, without regard for cueing-are identified. Second, in each string the effects of cueing are assumed to be limited to the highest-level chunk that contains the cue item. As a result, the part of each cued trial that involves traversal of the cued subtree is not used in the search for structure; only the latter parts, those involving natural traversal of the noncued subtrees, are used to build a second tree whose subtrees have the detailed structure induced from the noncued traversals. It follows that only noncued trials may be examined for the directionality of the root. ${ }^{6}$

${ }^{5}$ An ordered tree is selected as the primary representation of a chunk lattice because it fits some current ideas about chunk structures and the way they might be traversed to produce the order of recall. There are, of course, other ways to represent the chunking patterns revealed by our analysis, such as the set of rewriting rules, or productions, of a formal grammar. The program that embodies our algorithm can be obtained from the authors, in either RATFOR or FORTRAN versions.

${ }^{6}$ Cued trials are additionally used to indicate further partitioning of unidirectional chunks where appropriate. Undisrupted (noncued) recall of unidirectional chunks provides no information about where internal chunk boundaries are; we cannot infer boundaries without differences in recall order. However, we can (and do) induce further partitioning of unidirectional chunks from the regularities in the cued portions. Thus, final ordered tree representations are constructed by joining inferences about nested unidircctional chunks from cued portions to normal inferences about structure and directionality from noncued portions. 
As an example of the operation of this algorithm, assume that the following strings represent five trials of a subject's recall of the items $\mathrm{A}-\mathrm{H}$, organized in an unknown ordered tree. Though not illustrated here, every item should be used as a cue at least once, and a number of noncued trials should be interspersed. In this brief example, the italicized items indicate the cue on a cued trial:

$$
\begin{array}{lllllllll}
\text { 1. } & A & \text { B C D E F G H } \\
\text { 2. } & F \text { D E H G A C B } \\
\text { 3. } & H \text { G C B A D E F } \\
\text { 4. } & \text { G H D E F B C A } \\
\text { 5. } & \text { C B A D E F H G }
\end{array}
$$

In these strings, the largest nonoverlapping chunks (the subtrees of the root) are $A B C, D E F$, and $G H$. That is, the tree induced by all five trials, ignoring cueing, has the structure $((\mathrm{A}(\mathrm{BC}))((\mathrm{DE}) \mathrm{F})(\mathrm{GH}))$. Having identified the subtrees of the root, we assume the effect of cueing to be limited to the traversal of the cued subtree, and use only undisrupted traversals to build fine structure for each subtree. To further analyze ABC, we look only at Trials $2-5$, since Trial 1 was cued with a member of the $A B C$ subtree. In these trials, we find chunks $B C, A, B$, and $C$. To further partition DEF, we ignore Trial 2 (since it contains a cued instance of this chunk), and find DE, EF, D, E, and F. Examining Trials 1, 2, 4, and 5, we find $\mathrm{GH}$ partitioned simply into $\mathrm{G}$ and $\mathrm{H}$. The complete lattice for these chunks is shown in Fig. 2.

Converting this lattice to an ordered tree illustrates the correspondence that can be shown to exist between chunk lattices and ordered trees. After deleting the empty chunk we can see that, although parts of this lattice already have the form of a tree, it also contains cycles formed by overlapping chunks, such as DE and EF. However, the common inclusion of $E$ in both $D E$ and $E F$ simply indicates that $E$ must lie between $D$ and $F$ in every recall order. Likewise, the inclusion of DEF in both ABCDFF and DEFGH implies that DEF always occurs between $A B C$ and GH. Examination of the actual recall strings reveals the kind of directionality: DEF is

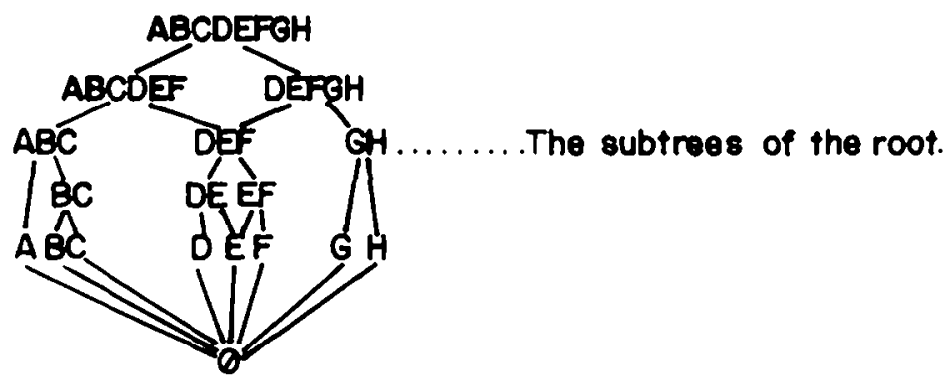

F1G. 2. Lattice display of the chunks found in the example recall strings. 
unidirectional, while the root node is bidirectional. The resulting ordered tree is that shown in Fig. 1. The expression for the tree of Fig. 1 is $\langle(\mathrm{A}(\mathrm{BC}))[\mathrm{DEF}](\mathrm{GH})\rangle$.

The amount of organization. Accompanying this initial work was the development of a measure of the amount of organization in an ordered tree. Previous efforts to attach a number to the amount of organization in free recall have typically considered only the number of strictly ordered groups occurring over trials, and thus have ignored the multilevel nature of the structure and the variety of ordered regularities possible (e.g., Tulving, 1962; reviewed by Shuell, 1969; but see Pellegrino, 1971, who recognizes the variety of directionality possible). Obviously, many aspects of an ordered tree may be used to describe its organization: its depth, the average size of the lowest level chunks, the average number of branchings at each level, to name a few. Moreover, different aspects may predict different behaviors related to organization (e.g., learning difficulty, long-term retention). At the outset, however, we have adopted a measure that seems to describe at least one important aspect of an ordered tree: the amount of variety the tree allows in the production of recall orders. The more structure there is, the less variation the subject has shown in recall, and the smaller is the set of recall strings that follow from that structure.

Our measure of the amount of organization in an ordered tree is the PRO, or "possible recall orders." It is the natural logarithm of the number of different recall orders that can be obtained by traversal of a given structure, or, alternatively, of the number of recall orders that contain its chunks. The extreme range of the untransformed values makes logarithms convenient. If $n$ items are completely unstructured, the PRO is $\ln (n !)$. If they are completely organized into a single, unidirectional chunk, the $P R O$ is $\ln (1)$. In calculating the PRO, each node of the tree is examined in turn, and a count is taken of the number of different ways its constituents can be ordered. The PRO is then the natural logarithm of the product of the counts from all the nodes. For example, the tree of Fig. 1 has two possible orders from the top node, 2 ! from node 2,2 ! from node 5 , one from node 3 , and 2 ! from node 4 . The PRO of this tree is $\ln (2 \times 2 ! \times 2 ! \times 1 \times 2 !)=\ln (16)$, i.e., there are exactly 16 strings over the items $\mathrm{A}-\mathrm{H}$ that contain the chunks of the lattice of Fig. 2. We use the PRO both as a descriptor of ordered trees and as an essential tool in the procedure we use to identify errors, described next.

Detecting errors. Our algorithm is extremely sensitive to errors, such as might occur when a subject intends to recall a particular item next, but temporarily forgetting it, continues his recall omitting that item, then later tacks it on to the end of the recall string. Just one inadvertent change in the position of an item will prevent the algorithm from detecting its true 
association to a chunk. Since people do occasionally make such errors, and since we wish to accurately represent structure in the face of errors, some procedure is necessary for detecting the rare misplacement of items.

The goal is to identify any recall trial that is very unlike the others, in the sense of implying a structure very different from that induced from the others-an "outlier" recall string. The procedure we have adopted is a variant of the general data-analysis procedure called "jackknifing", (Mosteller \& Tukey, 1977), which involves systematic repeated analyses of a data set in which one element is left out at a time. To understand our use of this procedure, imagine that in a set of trials, one trial includes the misplacement of an item or group of items. If the algorithm induces a tree from all trials, that anomalous trial will prevent the identification of the chunk or chunks that it alone does not contain. Suppose, then, that a tree is induced for each subset of the recall trials, excluding one trial at a time. The tree that is induced when the error trial is excluded will be much more structured than the others. The chunk that was hidden by the error will now appear, and the resulting tree will have a PRO that is markedly lower than the PRO of the other trees (those that result from the deletion of other trials). Thus, if the PRO of a tree induced in the absence of a particular trial is significantly lower than that of the others, that trial is identified as atypical and excluded from further analysis. As is usual in jackknifing, significance is determined by assuming that the PROs of all such subset trees are random and independent events, and using a $t$ distribution to determine confidence limits for the mean PRO value.

This procedure is very efficient for finding errors that occur only once, and can find all one-trial aberrations. Though it will not label as an error any anomaly that characterizes more than one trial, we considered this a small fault. If an error occurs twice, perhaps it should not be labelled an "error."

\section{Validation}

Three steps have been taken toward validating this technique for inducing ordered trees from free recall: In Experiment I, subjects were taught a particular organization of 24 words. From their free recall orders, the tree for each subject was induced and compared to the organization taught. In the same experiment, the time at which each item was recalled was recorded and then examined for correspondence to predictions from a simple model of recall timing from the tree. And, in Experiments II and III, free recall data were analyzed in domains in which there was no experimenter-defined organization, and the resulting trees were examined for face validity. In Experiment II psychology undergraduates and their instructor recalled course keywords, and in Experiment III computer experts and novices recalled computer language keywords. 


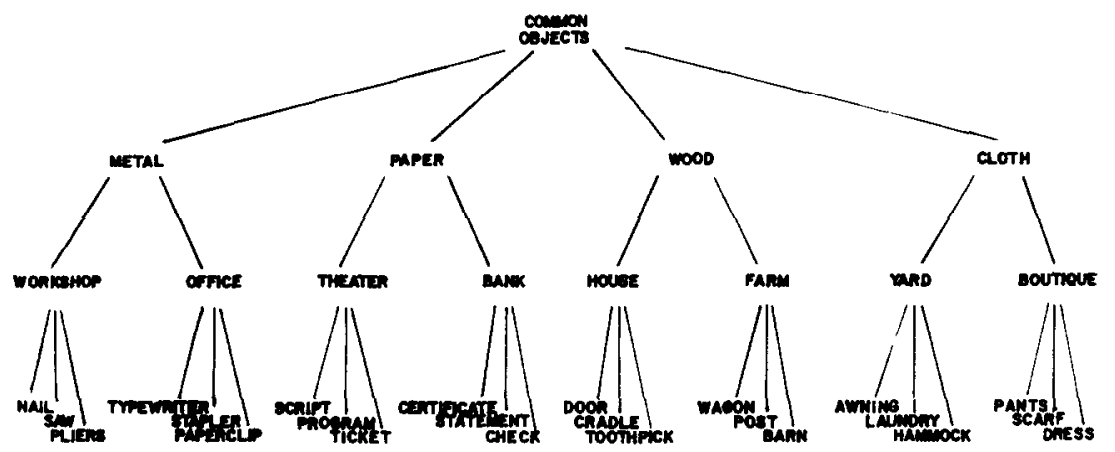

Fig. 3. Hierarchical arrangements of words from Experiment I.

\section{EXPERIMENT I}

\section{Comparing the Induced Structures to a Structure Known a Priori}

To assess the validity of this technique, we examined the free recall data from 20 subjects who were taught a particular organization of 24 words, an organization in which the words were grouped into threetuples, and the three-tuples into six-tuples. The words were displayed to the subject as shown in Fig. 3. Subjects were told to learn only the words at the bottom of the hierarchy, and that the labels at the nonterminal nodes were presented only for clarity. Subjects were asked to learn the words at their own pace, alternating study-test trials at will. When they had successfully recalled the 24 words correctly on two trials in a row, the formal part of the data collection began. At this point, subjects were asked to recall the 24 items 24 times, beginning each trial with the cue word and those that "go with it."

Each word in the set served as the cue word on one trial; on each trial a different word in the set served as the cue word. In this experiment, no noncued trials were given, so there is no direct evidence for directionality at the root of the induced trees. For this reason, all structures in Experiment I are conservatively represented with nondirectional roots. The vocal responses were tape-recorded for later transcription of both recall strings and interresponse times.

The recall strings from each subject were first "jackknifed" to eliminate errors, then analyzed into an ordered tree. For these 20 subjects, jackknifing revealed an average of 2.4 trials per subject (a range of 0-4) that were significantly unlike the rest, indicating an error.

The 20 induced structures correspond remarkably well to the structure presented, with two common, minor kinds of variations: Some of the structures show that presented three-tuples were not combined into sixtuples (i.e., subjects were not using the "material" dimension-wood, 


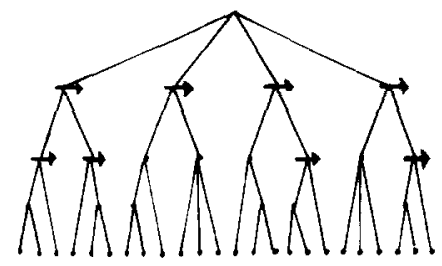

Subject $* 13$

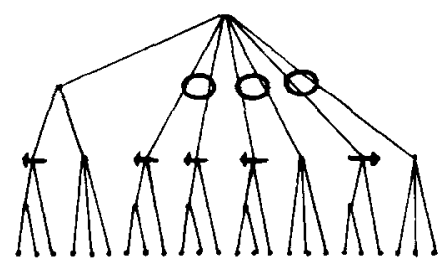

Subject 5
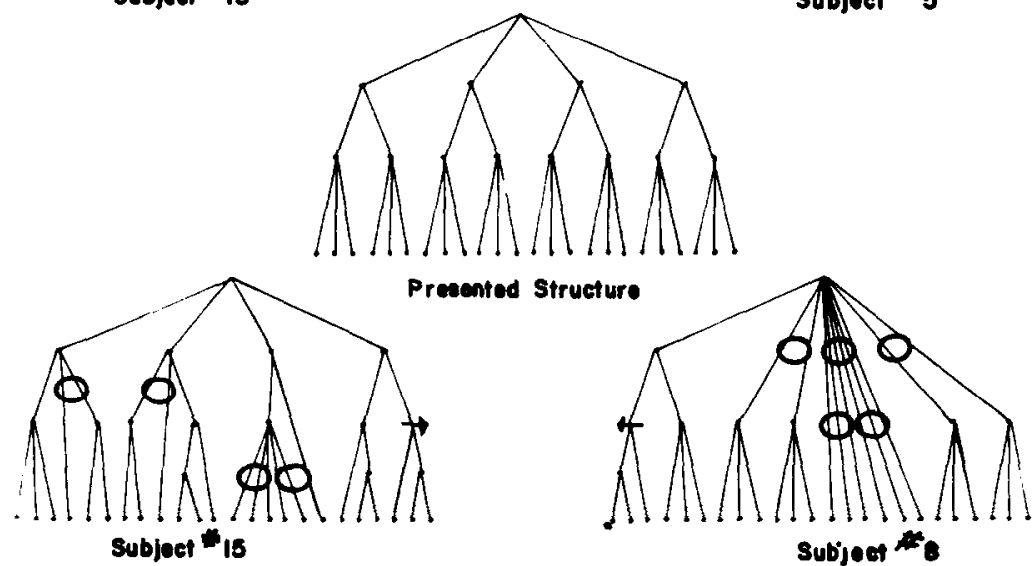

FIG. 4. Four subjects' organizations and the presented organization in Experiment I.

cloth, metal, or paper), and many included additional directionality where the presented organization was, of course, not explicitly directional. The presented structure, and the induced trees of four representative subjects are shown in Fig. 4. The structures are displayed here without labeling the terminal items, since the correspondence among trees was so high that the terminals on the induced trees could be ordered as in Fig. 3. Occasional exceptions involve only idiosyncratic ordering within unidirectional three-tuples.

The four trees shown represent the range of structures obtained. Subject 13 is typical of 7 of the 20 subjects; his structure corresponds to the presented organization with the addition of some directionality and further chunking within three-tuples. The other three trees span the variety of trees of the other 13 subjects. Subject 5 organized the items into three-tuples, but systematically avoided joining them into six-tuples. (Ovals in these figures indicate the "joins" that are missing.) Subject 15, on the other hand, organized some items into the six-tuples, but did not chunk within the six-tuples. Subject 8 , our worst subject, showed a mixture of extra partitioning, some directionality, a loss of six-tuple joins, and a complete "bushing" of one six-tuple. The PRO of the presented structure is 20.3 ; those for the 20 induced trees ranged from 0.7 to 27.8 , with a median of 13.3 . 
The correspondence between the presented tree and those induced for the subjects was assessed in two ways. The first involved simply counting the number of chunks in common. That is, if one subject grouped items $A-F$ as $((A B)[C D E] F)$ and the presented structure had been $([A B](C F(D E)))$, then chunks $A B$ and $D E$ are shared, but not $C D, C D E$, or CDEF. The presented tree has 12 nontrivial chunks (four 6-tuples and eight 3-tuples). The subjects' trees shared on average 10 of these (both mean and median) with a range of 6 to 12. In a second assessment of structural correspondence, we counted the number of each subject's recall trials that could have been produced by traversal of the presented hierarchy. For seven subjects, all the recall trials fit the presented structure. For the remaining subjects, $46 \%$ of the trials could not have been produced by traversal of the presented structure, but, of these, $82 \%$ broke only a single six-tuple join. The others broke individual three-tuples.

Converging evidence from recall timing. A commonly held view about how the structure of information affects the timing is that the time between recall of items within the same chunk should be shorter than the recall of items that are separated by chunk boundaries. This hypothesis is the basis of the Chase and Simon (1973a, b) technique for partitioning recall into chunks, and has been shown to be a strong correlate of organized recall in the work of Kellas, Ashcraft, Johnson, and Needham (1973); Murdock and Okada (1970); Patterson, Meltzer, and Mandler (1971); Pollio, Richards, and Lucas (1969); Pollio, Kasschau, and DeNise (1968); and Gelfand (Note 2). It seemed appropriate in this investigation, therefore, to examine interresponse times in recall to see if the induced hierarchical structure could account for systematic variations in the pauses in recall.

The timing of recall from these subjects was determined from the tape recording of the recall sessions. The two-track tape was slowed to halfspeed, and monitored by an assistant who then manually recorded a sharp tone on the second track whenever a word was uttered. This tape was then played at normal speed to a PDP-1 computer that measured the time in milliseconds between the tones on the second track. Since the times between recalls ranged from $300 \mathrm{msec}$ to $9 \mathrm{sec}$, the variability introduced by the assistant's reaction time in this transcription procedure was considered negligible. Because of the large range of times, we report times in centiseconds.

Figure 5 shows the mean interresponse times between successive recalls for each recall position. It is clear that subjects recalled items in three-tuples, pausing on average an additional full second between each three-tuple. This sort of summary description, however, averages over all the variety of structures the individual subjects produced, and gives no strong support for our particular technique. 


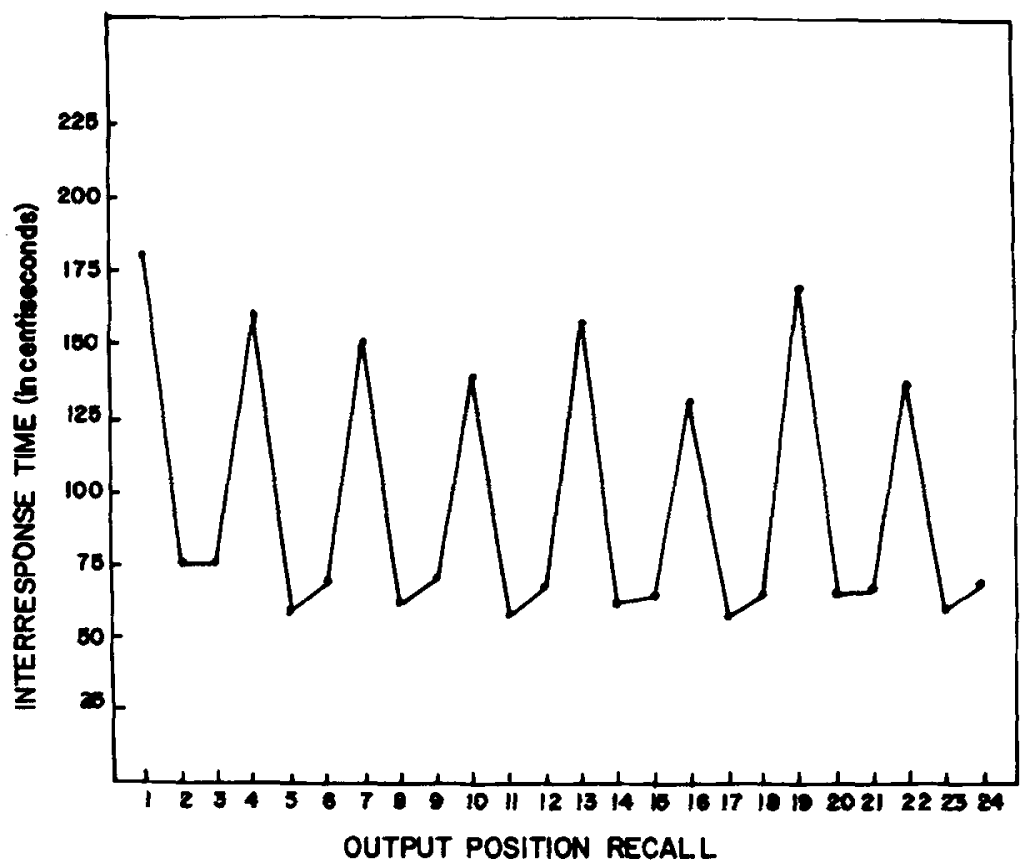

FIG. 5. Interresponse times at each output position in the recall strings.

Previous analyses of the pauses in free recall have typically compared only pauses between items within a chunk to pauses between items in different chunks. Our structures, however, are much richer and allow a much more detailed analysis. We can correlate times with the number of chunks boundaries crossed and the directionality of the chunks involved.

To examine pauses for our trees, we used a simple timing model. We posited that the greater the distance traversed in accessing the next item in the tree, the more time should be required for its recall. The distance between the two items was obtained by counting the number of nodes in the tree that separate them. For example, in the hierarchy in Fig. 1, B to C involves a distance of one, $C$ to $A$ two, and $C$ to $D$ four. Defining distance in this way allows a partition of all the interresponse times produced in the experiment ( $N=7500$ ) into categories that reflect the individual subjects' trees. The relationship between this measure of distance and average pause length is shown in Fig. 6. In general, as distance increases, pauses get longer. To assess the significance of this relationship, we fit a regression line to each subject's mean interresponse times as a function of the associated distances in his tree. The mean of the slopes of these regression lines over the 18 subjects having more than one distance is 18.6 csec/unit distance, a value significantly larger than zero, $t(17)=4.76$, $p<.01$. 


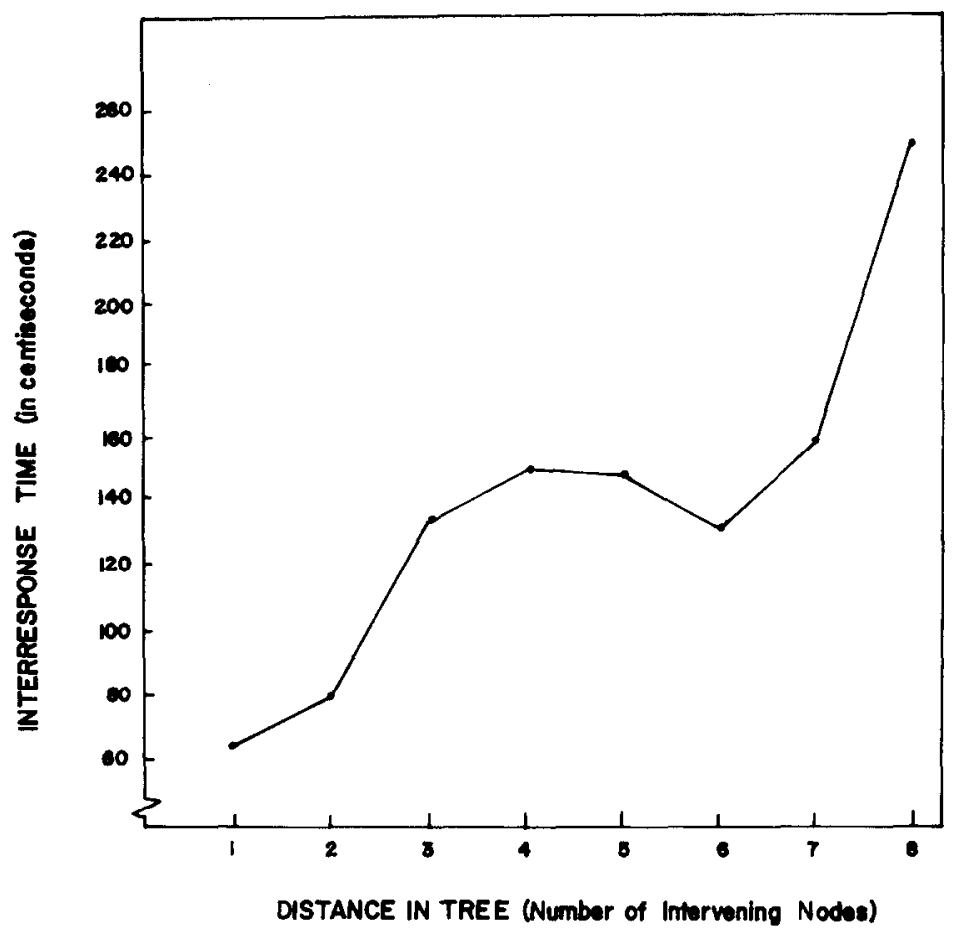

FIG. 6. Interresponse times as a function of distance traversed in the induced tree, distance being a count of the number of intervening nodes.

This significant increase in response time with simple tree distances, though encouraging, does not show the full extent of the relationship between the structure induced for an individual subject and that same subject's pattern of recall pauses. Figure 7 shows four trees with the average interresponse times written at the nodes. The numbers in the nodes are the averages of the interresponse times between items for which that node is the highest node to be traversed between them. For example, Subject 8 took on average $45 \mathrm{csec}$ in recalling $C$ after $B, 70 \mathrm{csec}$ recalling $B$ after $A, 86 \mathrm{csec}$ recalling $D$ after $C$, and $116 \mathrm{csec}$ recalling $J$ after $C$. This figure shows the structures and times for the four subjects described earlier. In general, it demonstrates a good (but not perfect) correspondence between structure and time: the larger the distance between two items, the longer the pause separating them.

To analyze the significance of the correspondence between these interresponse times and tree structures, we first annotated each subject's tree with the times, as shown in Fig. 7. If times are related to these structures as predicted, then there should be an order relationship between the times at the various nodes. For example, for Subject 8 , the node representing 

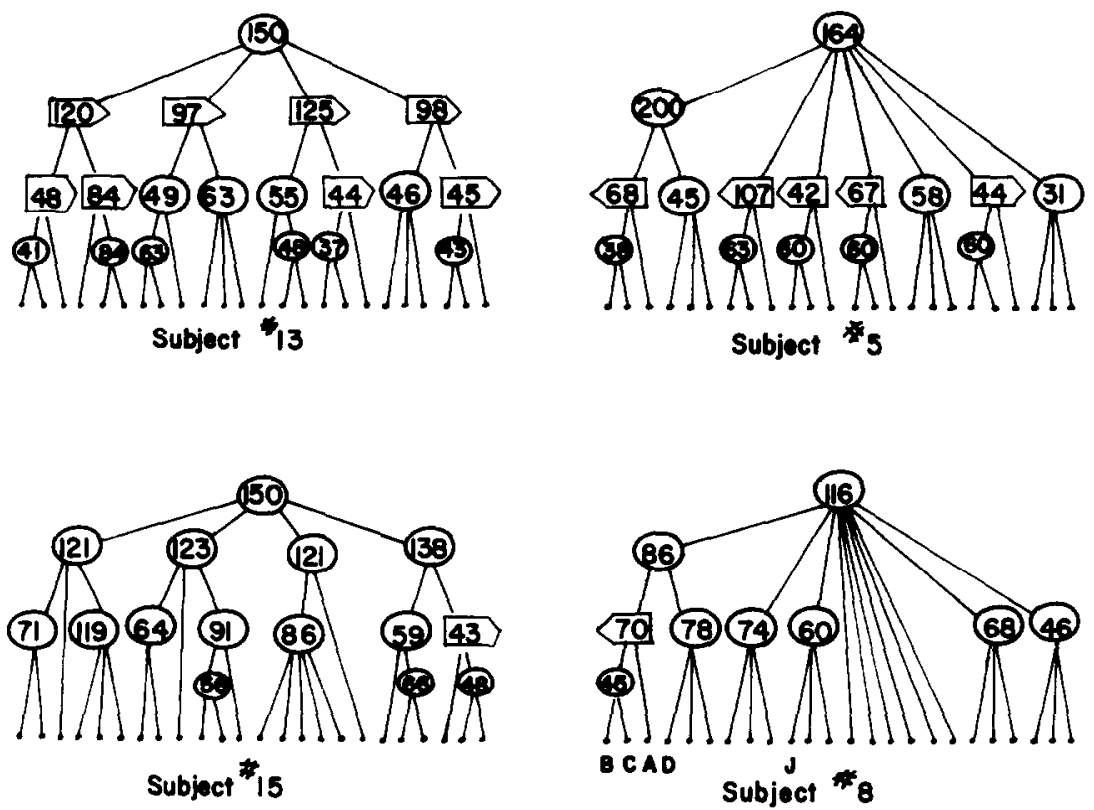

Fig. 7. Interresponse times superimposed on four subjects' ordered trees. Each time listed is the average of those times between items for which that node is the highest node traversed between them.

recall from $B$ to $C$ has, as predicted, a smaller mean interresponse time than that from $B$ to $A$, which involves traversal through more nodes. Thus, the order relation between the times shown at these two nodes fits the prediction. We counted the number of such order relations that fit the order relation predicted by the structure, including both adjacent and distant relations. To assess the significance of this statistic (called "Ord") within each subject, we estimated the underlying distribution by calculating Ord for 10,000 random assignments of the set of a subject's actual times to the nodes of his structure. The probability of the obtained Ords appearing by chance was estimated to be less than .05 for 16 of the 20 subjects (less than .01 for 9 of these) and less than .10 for the remaining 4 .

One central feature of the technique is the identification of consistent recall directionality. The question arose in the analysis of pauses, then, whether that directionality had any corresponding evidence in timing. Because directionality is found at widely different points in the 20 subjects' structures, the analysis reported here is restricted to pauses only within the kind of substructure that every subject had, an induced threetuple. Table 1 lists the average pauses within induced three-tuples for the different kinds of three-tuple organization evidenced. Subjects are slower in recalling from a nondirectional three-tuple than from a unidirectional 
TABLE 1

Pause Length as a Function of the Kind of Partitioning Within a Three-Tuple

\begin{tabular}{llll}
\hline Kind of three-tuple & \multicolumn{1}{c}{ Average pause } & PRO \\
\hline $\begin{array}{l}\text { Nondirectional: } \\
\text { Three-tuple }\end{array}$ & $(\mathrm{XXX})$ & $(74)$ & 1.8 \\
$\begin{array}{l}\text { Subpartitioned } \\
\text { Unidirectional: }\end{array}$ & $((\mathrm{XX}) \mathrm{X})$ & $((63) 92)$ & 1.4 \\
Partitioned with & $([\mathrm{XX}) \mathrm{X})$ & $([58] 67)$ & .7 \\
nondirectional & {$[\mathrm{X}(\mathrm{XXX)})$} & {$[63(65)]$} & .7 \\
& {$[(\mathrm{XX}) \mathrm{X}]$} & {$[(46) 46]$} & .7 \\
& & & \\
Partitioned with & {$[\mathrm{X}[\mathrm{XX}]]$} & {$[60[44]]$} & 0 \\
nested unidirectional & {$[[\mathrm{XX}] \mathrm{X}]$} & {$[[48] 50]$} & 0 \\
& {$[\mathrm{XXX}]$} & {$[49]$} & 0 \\
\hline
\end{tabular}

one, $t(22)=4.19, p<.005$, where the data for the $t$ test were the means of interresponse times for particular three-tuples for particular subjects. Directionality does have a correlate in timing.

\section{EXPERIMENT II}

Structures of Course Keywords for Students and the Instructor

This experiment and the next demonstrate the face validity of the induced trees, and illustrate the variety of materials that can be accommodated by the technique.

On the first day of an introductory psychology class, students and the instructor were given a list of 16 words to memorize in any order they wished. The words were psychology keywords that had technical meaning for the instructor, but had either a normal, nontechnical meaning (e.g., "staircase") or no meaning (e.g., "Mach") for the students. After the subjects had memorized the list, they produced one complete, noncued recall each day for 10 days (illustrating a second way to induce variety, or reduce stereotypy). Figs. 8, 9, and 10 show three illustrative trees from the 20 class members. The instructor's organization (Fig. 8) appears to be based on the terms' underlying technical meanings. For example, "hue" and "saturation" are dimensions of color, "rank" and "mode" are statistical terms, etc. Subject 3's structure (Fig. 9) is an example of a loosely alphabetic organization; it is similar to those of about half the 20 students. Alphabetizing is an appropriate mnemonic strategy for unorganized items, and indicates that while these students are sophisticated learners, they know little about the words' underlying organization. It is also evident that the degree of organization, per se, is not to be equated with expertise, since some of these Naive subjects have highly structured 


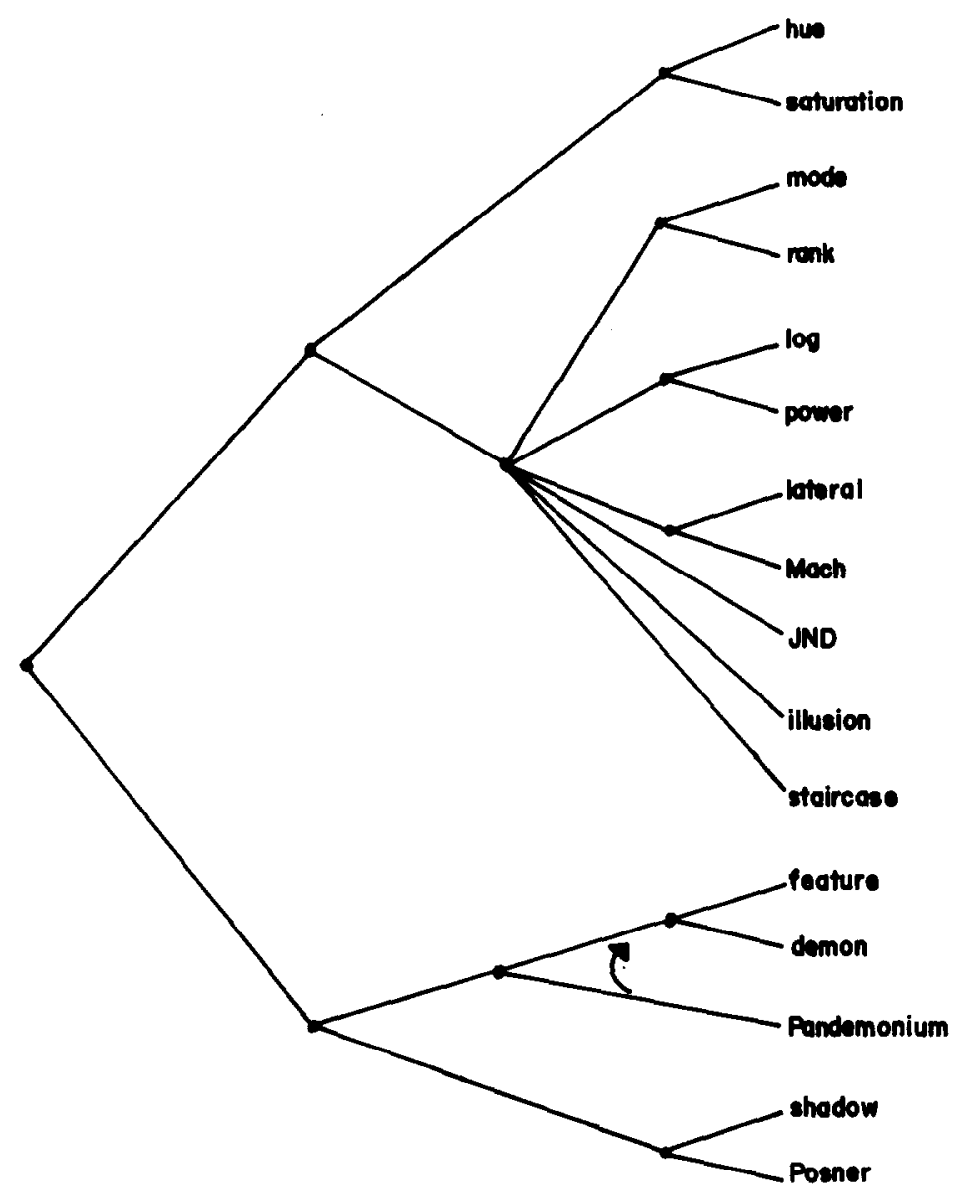

FIG. 8. Instructor's organization of psychology course keywords.

trees. Subject 8 (Fig. 10) illustrates the unorganized "bushiness"' of most of the remaining trees, with one striking exception. Ths student appears to know something about both the Pandemonium model of pattern recognition ("Pandemonium," "feature," and "demon" cluster) and lateral inhibition as an explanation of Mach bands ("Mach" and "lateral" cluster).

\section{EXPERIMENT III}

Structures of Computer Reserved Words for Expert and Novice Programmers

This study, part of the work of McKeithen (Note 4), illustrates an application of this technique to characterizing differences in expert-novice knowledge structures. 


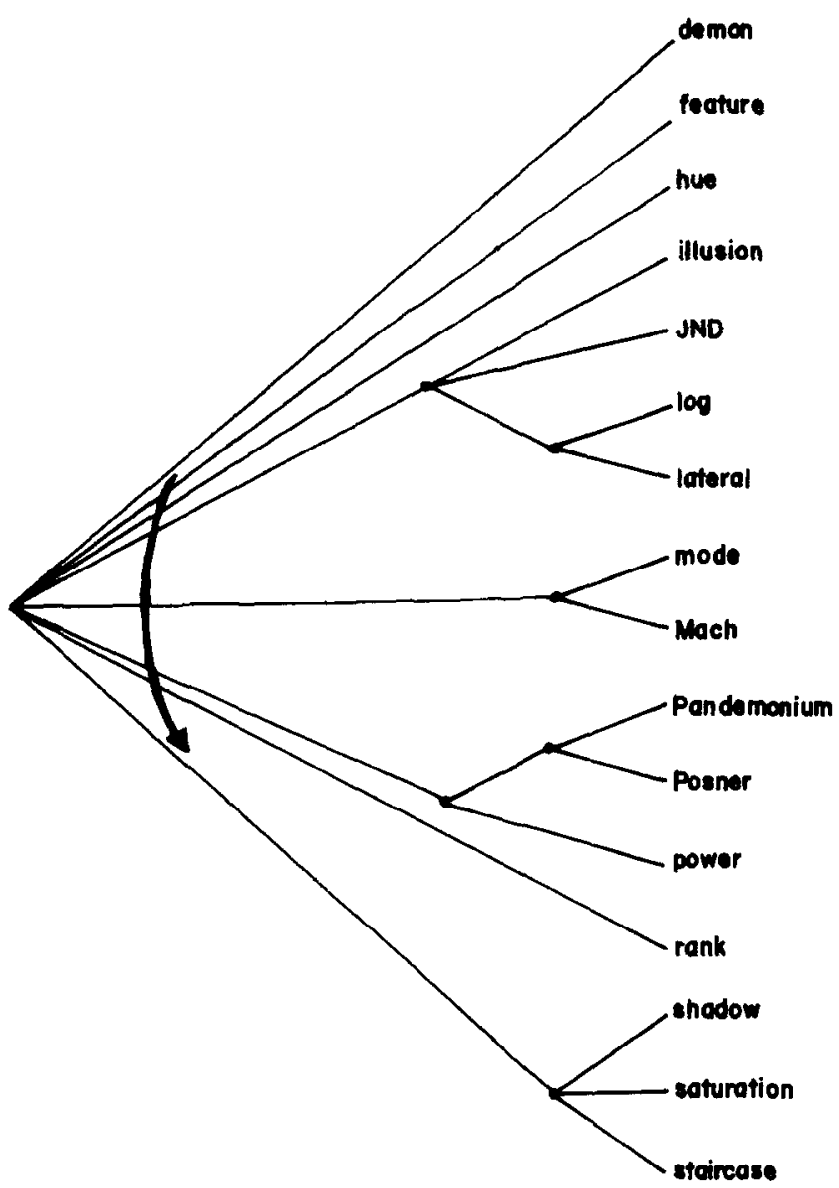

FIG. 9. Student's alphabetic organization of psychology course keywords.

McKeithen was concerned with the differences between expert and novice programmers, and focused on the way they use their knowledge to understand an unfamiliar program. She asked ALGOL W Experts, Novices (students just finishing their first ALGOL W course), and Naives (students enrolled in an ALGOL W course, but tested on the first week of class) to reproduce a short program after a series of 2-min presentations. Experts not only did better than the others, as expected from past work on chess, Go, and electronics experts (Chase \& Simon, 1973a, b; deGroot, 1966; Reitman, 1976; Egan \& Schwartz, 1979), but also organized their recall around lines of the program that contained related ALGOL W keywords. To further examine the structure of the associations among these keywords, McKeithen had her 22 subjects repeatedly recall 21 of ALGOL W's keywords (the "reserved words," those that cannot be used 


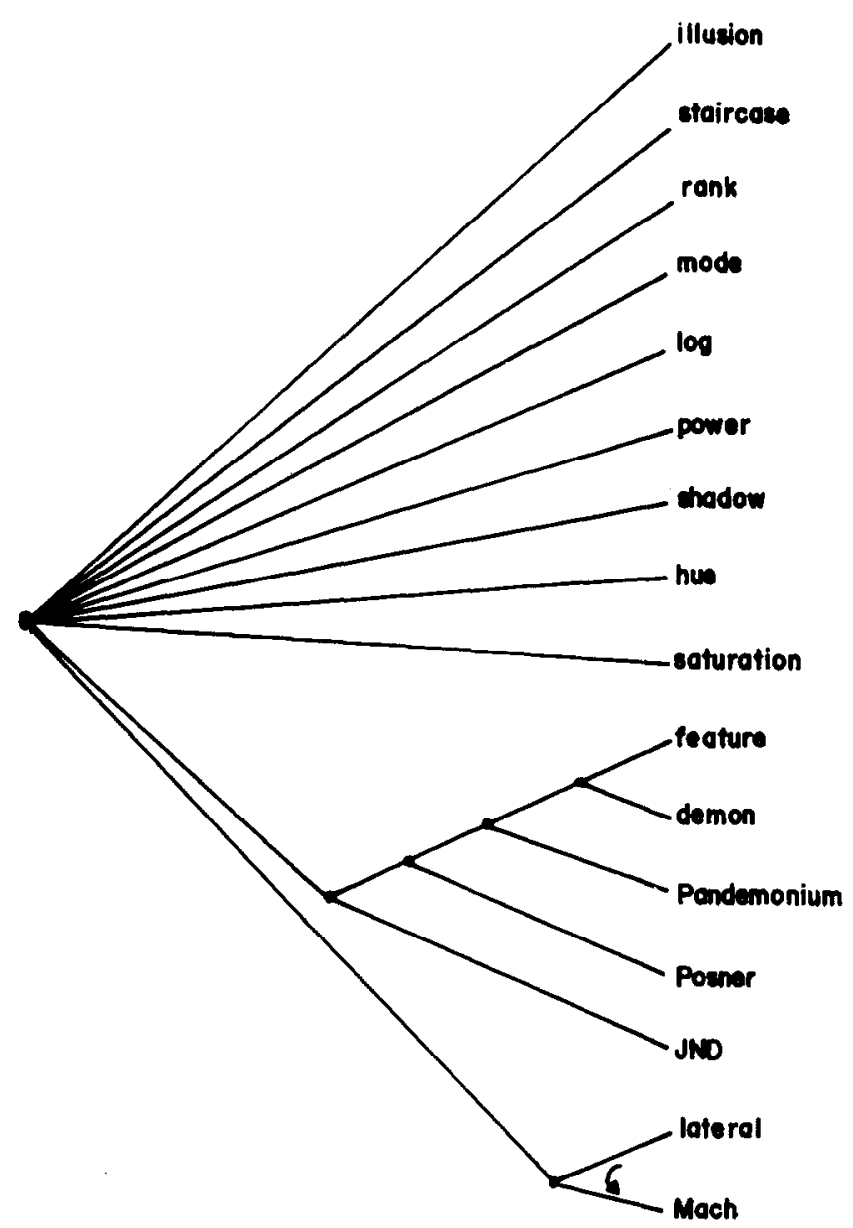

Fic. 10. Student's bush-like organization with clustering of words related to Pandemonium and Mach bands.

by a programmer as variable names). ${ }^{7}$ Each subject recalled the 21 items 25 times. Four of the trials were not cued, and each keyword served as a cue on one trial, as in Experiment I. Each subject's recall strings were then converted into an ordered tree.

The trees of one Naive subject and one Expert subject are shown in Figs. 11 and 12. They illustrate the general pattern of results: Experts tend

${ }^{7}$ Since interresponse times were to be measured, only the one-syllable reserved words were used. Four other words, ABS, DIV, SHL, and SHR, which might be considered one-syllable, were not included in the set because pronunciation difficulties might have adversely affected the times. As a result, such chunkable words as END appear unassociated with any chunk because their natural chunk-mates, e.g., BEGIN, were eliminated from the set as two-syllable words. 


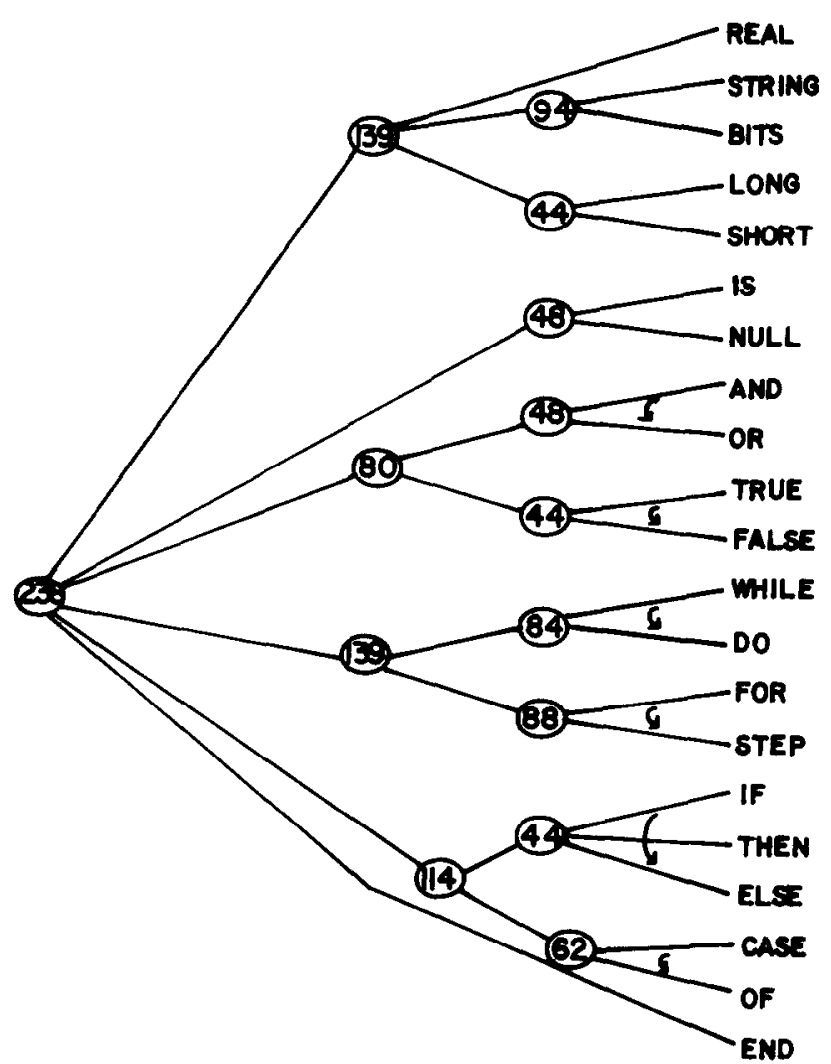

FIG. 11. Expert's organization of ALGOL $W$ reserved words with interresponse times noted at each node.

to organize keywords according to their functional meaning, for example, grouping related concepts like WHILE-DO and FOR-STEP according to their function in iterations. Naives, on the other hand, though they have as many groups as Experts (the mean PRO for Experts is 6.79; for Naives it is 7.92) seem to base them on common-language associations rather than on computer functions. Furthermore, Experts were similar in their patterns of organization, while the Novices and Naives organized the words more idiosyncratically. One way to see this is to count the number of chunks in common for all members of a skill level, as in Experiment I. Neither Naives nor Novices shared any chunks. The Experts, on the other hand, all produced chunks containing TRUE-FALSE, CASE-OF, and THEN-ELSE. Since there were no commonalities within either the Novice or Naive groups, there could be none across groups.

Though there were no chunks common to all subjects, there were some 


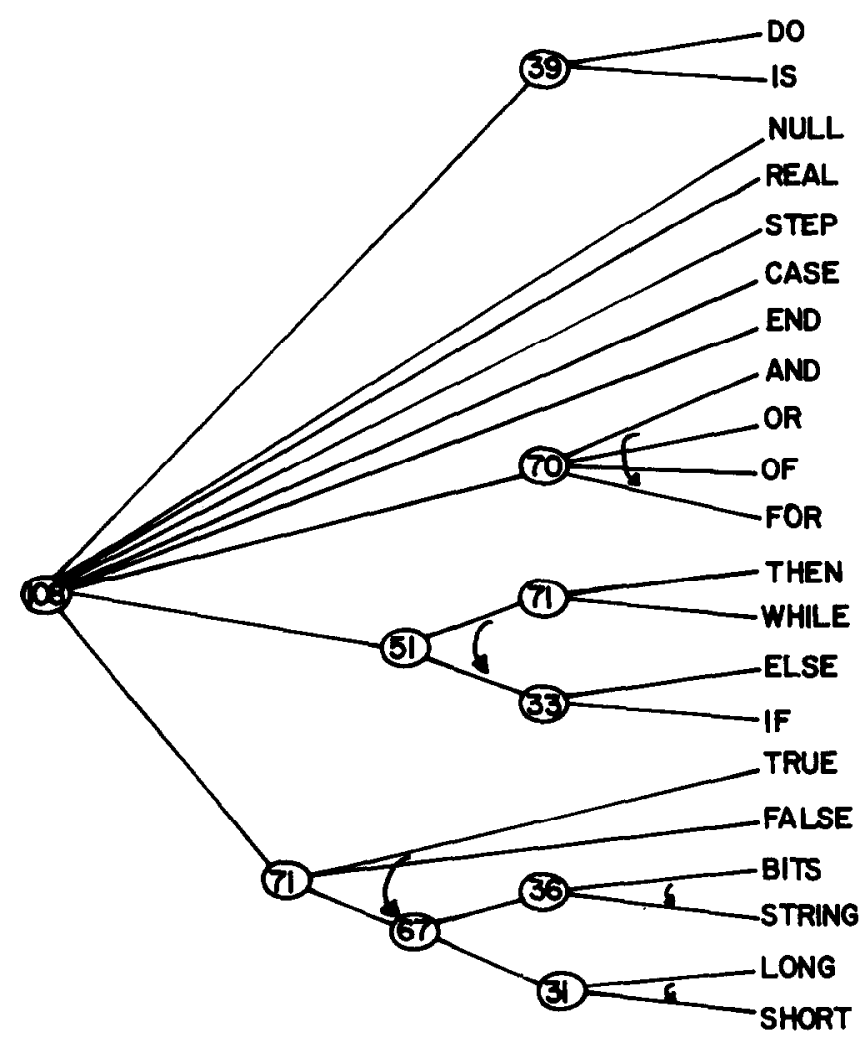

Fig. 12. Naive subject's organization of ALGOL $W$ reserved words with interresponse times noted at each node.

common to many subjects. Common language associations led many of the subjects, regardless of level, to cluster LONG-SHORT and TRUE-FALSE. Associations specific to ALGOL $W$, such as STEP-FOR and CASE-OF, were clustered by most of the Experts and Novices, but never by the Naives.

This study also provided additional evidence for a relationship between structure and interresponse times. As in Experiment I, recall was tape recorded and transcribed into pauses. The structures in Figs. 11 and 12 are annotated with the average pauses at the nodes, using the procedure described in Experiment I. Inspection of these figures shows an excellent correspondence between the pauses and the induced structures. Of the 15 subjects whose times were collected and whose structures were neither wholly bushes nor single unidirectional chunks, four had Ords significant at less than .05 , and six more with too few nodes to possibly reach significance had all order relations in the predicted direction. Of the remaining five subjects, three were close to significance, having only one violation 
of the predicted order (Ords were 4/5,7/8, and 7/8), and two were not significant (12/17 and 2/5). In general, as in Experiment I, interresponse times were clearly correlated with distance in the induced trees.

\section{DISCUSSION AND SUMMARY}

This work shows that the task of recalling known items many times from many different starting points can reveal meaningful facets of the underlying organization. Since the task of recalling a large number of items is difficult, people are impelled to use any underlying associations they may have to aid them. And, the resulting recall regularities reveal some of that organization of information. Moreover, as these two latter studies show, this technique has the potential for diagnosing the kinds of organization of knowledge students have when they enter a field of study, and might be useful in assessing the growth of their knowledge as they progress toward the expert level.

One unique aspect of this technique is its identification of directionality between chunks at all levels in a hierarchy. The value of this identification appeared at three points in the studies presented here. First, pauses were significantly shorter for ordered as opposed to unordered three-tuples (Experiment I). Second, without knowledge of directionality, it would have been impossible to label the students' organizations in Experiment II as "loosely alphabetical." Third, in Experiment III both Expert and Naive computer programmers chunked DO and WHILE, but differed in the order in which they were recalled. For Naive subjects, the order [DO WHILE] reflects common language usage, whereas the Expert order, [WHILE DO], is clearly based on the natural order in ALGOL W iteration statements. Directionality appears frequently in free recall; our ordered trees capture and display it at many levels.

One limitation of this technique involves directionality. Though we can correctly identify chunk directionality, we do not identify more general precedence relationships. For example, in our analysis, the recall orders $\mathrm{ABC}, \mathrm{ACB}$, and $\mathrm{CAB}$ produce a bush, $(\mathrm{ABC})$, that does not reveal that $\mathrm{A}$ always preceded $B$. Our algorithm ascribes directionality only to contiguous chunks and ignores such loose precedence relationships.

A second unique aspect of the technique is that it produces detailed organization that is highly reliable. Any chunking or ordering identified is present in the noncued portion of all trials. There are, of course, drawbacks to this requirement of explicit reliability. Though jackknifing can identify single-trial errors, it cannot identify pairs of identically erring trials (unless one also systematically deleted all pairs of trials, etc.). And, one should not infer from a bush that there is no organization. Bushes could result from either a different kind of recall rule or a different kind of memory structure, as described below. 
We have shown here that our technique provides reliable, interesting descriptions of some of the regularities in recall and represents them as an ordered tree. The conclusions that could follow from this work are at two levels: The first conclusion involves the technique only as a descriptor of recall regularities; the second involves inferences about the true underlying mental representation of organized information and the recall processes that operate on it.

The technique does describe regularities that appear in multitrial free recall. The description involves specification of the chunk contents, their hierarchical nature, and directionality of recall where appropriate. Is an ordered tree a useful representation of these kinds of regularities in recall? First, we have achieved success in applying this technique to recall; we revealed a great deal of structure. This supports the common conception that information is stored in some format akin to chunks and that people recall them with some regularity. This regularity must be explained by whatever theory of recall and memory that we end up with.

Second, a description of this kind lends itself well to the examination of questions relating to other measurable aspects of recall. For example, what recall process produces the distribution of pauses in recall of organized material? What determines the ease of learning another related organization? And, how are judgments of similarities affected by different kinds of underlying organization? Having reliable, detailed descriptions provided by ordered trees serves as a platform from which to begin to ask these specific questions.

The second conclusion, dealing with whether the true underlying organization is an ordered tree or not, is tenuous at this stage of our investigation. That we found a great deal of the kind of structure we were looking for does not confirm our model of recall. Some other kind of process operating on another kind of structure might produce the orders of recall and the kinds of regularities that our ordered trees summarize. A probabilistic grammar is an example, albeit one that is so closely related to tree traversal as to be almost indistinguishable. Or, recall could be produced by some kind of regular retrieval process operating on a matrix (Broadbent, Cooper, \& Broadbent, 1978) or a network. We rely on converging evidence (e.g., pauses, ease of transfer to a new structure, etc.) to test the reality of this kind of representation, and will continue with ordered trees until we can find another model of recall on another memory structure that accounts for the regularities observed here and more (e.g., accounting not only for chunks, directionality, and hierarchical inclusion, but loose precedence relationships as well).

As a descriptor, this technique has application to tasks and topics outside those of recall from memory, most notably to topics in decision making and perception. It can summarize regularities in a set of linear 
strings of any objects. In the work reported here, these strings are assumed to be generated in recall by traversal of a hierarchy in memory. But, any time a set of items is repeatedly ordered (by either a single subject or many subjects), and the chunking and ordering of these data are of theoretical interest, the technique is applicable. For example, within the area of decision making, when people rank their preferences for a set of objects, they typically are inconsistent. This inconsistency suggests that the underlying representation of these preferences is not a linear order, but an ordering on equivalence classes. (For the classic exploration of preference orders, see Coombs, 1964.) If we ask a subject to produce a set of preference orders and the orders differ from each other, an ordered tree of preference classes could be induced.

Similarly, in perception, the strings could be derived from reproduction of a visual pattern (much like the "Perception task" of Chase \& Simon, 1973a, b; and Reitman, 1976; in their examinations of perception of game positions). From the orders in which the constituents of the pattern are produced, it would be possible to induce the structure of the perceptual chunks.

In summary, the technique introduced here induces the organization of information in memory from systematic inspection of regularities in ordered strings, here the order in which items are produced in free recall. The representation of this organization takes the form of an ordered tree. The technique has the advantage of being based on a theory of the way in which the data were generated, and can be shown to produce a unique structure that captures all of the kinds of regularities the theory of recall prescribes. Also presented is a collateral technique for measuring the amount of organization evidenced in a structure, together with a procedure for identifying errors. The experimental work has demonstrated (1) an ability to recover the details of an organization presented to subjects and (2) converging evidence for the structures induced from the pattern of recall pauses. In addition, the technique's application to structure unknown a priori was shown to produce organizations that were easy to interpret and pauses that further confirmed the details of the induced structures.

\section{REFERENCES}

Arnold, J. B. A multidimensional scaling study of semantic distance. Journal of Experimental Psychology Monograph, 1971, 90, 2, 349-372.

Bower, G. H., \& Springston, F. Pauses as recoding points in letter series. Journal of Experimental Psychology, 1970, 83, 421-430.

Bower, G. H., \& Winzenz, D. Group structure, coding, and memory for digit series. Journal of Experimental Psychology, 1969, 80(2, Pt. 2).

Broadbent, D. E., Cooper, P. J., \& Broadbent, M. H. P. A comparison of hierarchical and matrix retrieval schemes in recall. Journal of Experimental Psychology: Human Learning and Memory, 1978, 4, 486-497. 
Bushke, H. Learning is organized by chunking. Journal of Verbal Learning and Verbal Behavior, 1976, 15, 313-324.

Chase, W. G., \& Simon, H. A. Perception in chess. Cognitive Psychology, 1973, 4, 55-81. (a)

Chase, W. G., \& Simon, H. A. The mind's eye in chess. In W. G. Chase (Ed.), Visual information processing. New York: Academic Press, 1973. (b)

Coombs, C. H. A theory of data. New York: Wiley, 1964.

Cunningham, J. P., \& Shepard, R. N. Monotone mapping of similarities into a general metric space. Journal of Mathematical Psychology, 1974, 11, 335-363.

deGroot, A. Perception and memory versus thought: Some old ideas and recent findings. In B. Kleinmuntz (Ed.), Problem solving. New York: Wiley, 1966.

Egan, D. E., \& Schwartz, B. J. Chunking in recall of symbolic drawings. Memory and Cognition, 1979, 7(2), 149-158.

Friendly, M. L. In search of the M-gram: The structure of organization in free recall. Cognitive Psychology, 1977, 9, 188-249.

Holman, E. W. The relation between hierarchical and Euclidean models for psychological distances. Psychometrika, 1972, 37(4), 417-423.

Jantz, E. M., \& Underwood, B. J. R-S learning as a function of meaningfulness and degree of S-R learning. Journal of Experimental Psychology, 1958, 56, 174-179.

Johnson, N. F. Organization and the concept of a memory code. In A. W. Melton, \& E. Martin, Coding processes in human memory Washington, D.C.: Wiley, 1972. Pp. 125-159.

Johnson, S. C. Hierarchical clustering schemes. Psychometrika, 1967, 32, 241-254.

Kellas, G., Ashcraft, M. H., Johnson, N. S., \& Needham, S. Temporal aspects of storage and retrieval in free recall of categorized lists. Journal of Verbal Learning and Verbal Behavior, 1973, 12, 499-511.

Kruskal, J. B. Multidimensional scaling by optimizing goodness of fit to a non-metric hypothesis. Psychometrika, 1964, 29, 1-27. (a)

Kruskal, J. B. Non-metric multidimensional scaling: A numerical method. Psychometrika, 1964, 29, 115-129. (b)

Martin, E., \& Noreen, D. L. Serial learning: Identification of subjective sequences. Cognitive Psychology, 1974, 6, 421-435.

McLean, R. S., \& Gregg, L. W. Effects of induced chunking on temporal aspects of serial recitation. Journal of Experimental Psychology, 1967, 74, 455-459.

Monk, A. F. A new approach to the characterization of sequential structure in multi-trial free recall using hierarchical grouping analysis. British Journal of Mathematical and Statistical Psychology, 1976, 29, 1-18.

Mosteller, F., \& Tukey, J. W. Data analysis and regression: A second course in statistics. New York: Addison-Wesley, 1977.

Murdock, B. B., Jr., \& Okada, R. Interresponse times in single-trial free recall. Journal of Experimental Psychology, 1970, 86, 263-267.

Patterson, K. E., Meltzer, R. H., \& Mandler, G. Interresponse times in categorized free recall. Journal of Verbal Learning and Verbal Behavior, 1971, 10, 417-426.

Pellegrino, J. W. A general measure of organization in free recall for variable unit size and internal sequential consistency. Behavioral Methods and Instrumentation, 1971, 3(5), 241-246.

Pollio, H. R., Richards, S., \& Lucas, R. Temporal properties of categorical recall. Journal of Verbal Learning and Verbal Behavior, 1969, 8, 529-536.

Pollio, H. R., Kasschau, R. A., \& DeNise, H. E. Associative structure and the temporal characteristics of free recall. Journal of Experimental Psychology, 1968, 76, 190-197.

Reitman, J. S. Skilled perception in Go: Deducing memory structures from inter-response times. Cognitive Psychology, 1976, 8, 336-356.

Sattath, S., \& Tversky, A. Additive similarity trees. Psychometrika, 1977, 42, 319-345. 
Shepard, R. N. The analysis of proximities: Multidimensional scaling with an unknown distance function. I. Psychometrika, 1962, 27, 125-140. (a)

Shepard, R. N. The analysis of proximities: Multidimensional scaling with an unknown distance function. II. Psychometrika, 1962, 27, 219-246. (b)

Shepard, R. N., \& Arabie, P. Additive clustering: Representation of similarities as combinations of discrete overlapping properties. Psychological Review, 1979, 86, 87-123.

Shuell, T. J. Clustering and organization in free recall. Psychological Bulletin, 1969, 69, $344-354$.

Tulving, E. Subjective organization in free recall of "unrelated" words. Psychological Review, 1962, 69, 344-354.

\section{REFERENCE NOTES}

1. Cunningham, J. P. Discrete representations of psychological distance and their applications in visual memory. Unpublished dissertation, University of California at San Diego, 1976.

2. Gelfand, H. Organization in free recall learning: Output contiguity and inter-response times as a function of presentation structure. Technical Report No. 29. Ann Arbor, MI: Human Performance Center, 1971.

3. Graesser, A. The memory for and comprehension of sentences. Unpublished doctoral dissertation, University of California at San Diego, 1976.

4. McKeithen, K. B. Assessing knowledge structures in novice and expert programmers. Unpublished doctoral dissertation, University of Michigan, 1979.

5. Vorberg, D. Maximum likelihood construction of hierarchies from recall orders in multi-trial free recall. Notes of a talk given at Rockefeller University recorded in a letter to Herman Bushke, 1973.

(Accepted December 19, 1979) 